\title{
ANALYTICAL MODEL OF NUCLEAR ENVIRONMENTAL EFFECTS WITH PIEZOELECTRIC WAFER ACTIVE SENSORS
}

\author{
Bin Lin, Lingyu Yu, Victor Giurgiutiu \\ University of South Carolina \\ Columbia, SC 29208, USA \\ linbin@sc.edu \\ Matthieu Gresil \\ University of Manchester \\ Manchester, UK \\ Adrian E. Mendez Torres \\ Savannah River Nation Laboratory \\ Aiken, South Carolina, USA
}

\begin{abstract}
The increasing number, size, and complexity of nuclear facilities deployed worldwide are increasing the need to maintain readiness and develop innovative sensing materials to monitor important to safety structures (ITS) for pipe and vessels (PVP) application. For example, nuclear dry cask storage system (DCSS) is a safety-critical facility in need of monitoring over prolonged periods of time. Technologies for the diagnosis and prognosis of PVP systems can improve verification of the health of the structure that can eventually reduce the likelihood of inadvertently failure of a component.

The past two decades have witnessed an extensive sensor technology development using permanently installed piezoelectric wafer active sensors (PWAS) for structural health monitoring (SHM). PWAS have emerged as one of the major SHM technologies developed particularly for generating and receiving acousto-ultrasonic waves for the purpose of continuous monitoring and diagnosis. Durability and survivability of PWAS under nuclear environmental exposures has been tested preliminarily. However, the analytical model of PWAS based sensor and sensing system has not been developed with adequate solutions and guideline. This paper presents a study on an analytical model of nuclear environmental effects with PWAS. Environmental variability of a Nuclear-SHM system includes changes in both the sensors and the sensing
\end{abstract}

methodology including acoustic emission (AE), guided ultrasonic waves (GUW), and electro-mechanical impedance spectroscopy (EMIS). We considered the environmental variability considers the effects of temperature changes and radiation. We superposed these changes on the analytical models and determined how much the structural sensing signals change is due to these environment effects for the DCSS system. The analytical modeling of various structural sensing methods to environmental disturbances was studied and the potential of PWAS as irradiation sensors for PVP applications was explored. The paper ends with conclusions and suggestions for further work.

\section{INTRODUCTION}

Many pressure vessel and piping (PVP) systems are safetycritical facilities in need of monitoring over prolonged periods of time. Structural health monitoring (SHM) is an emerging technology that aims at monitoring the state of a structure through the use of networks of permanently mounted sensors. SHM technologies have been developed primarily within the aerospace and civil engineering communities. However, SHM concepts and methodologies could be extended to other safetycritical systems. Many PVP applications, as for example, nuclear power plants (NPP) and nuclear dry cask storage systems (DCSS) are safety-critical facilities in need of 
monitoring over prolonged periods of time. One of the key aspects of improving the reliability, sustaining the safety, and extending the life of current PVP is to develop technologies that can better diagnose their state of structural health.

SHM is an emerging technology that uses in-situ sensory system to perform rapid nondestructive detection of structural damage as well as long-term integrity monitoring. The permanently installed (embedded) sensors can probe the structures at any time over the entire service life, which is superior to the handheld conventional ultrasonic nondestructive testing (NDT) techniques. Combined with appropriate data analysis algorithms, SHM can further provide timely information regarding the structural integrity for condition assessment and diagnosis of components important to safety (ITS) at any time. Hence, the integration of a nuclear SHM system into a nuclear spent fuel storage system will facilitate the evaluation of degradation and aging of used nuclear fuel containers and storage facilities over extended storage periods. Eventually, it will ensure a systematic methodology for assessing and monitoring nuclear waste storage systems without incurring human radiation exposure. The primary goal of in-situ sensing of structural components is to reliably interrogate large areas and detect structural anomalies. However, when applied to nuclear environment, even the relatively less harsh DCSS situation, prolonged exposure to nuclear radiation as well as elevated temperature may introduce measurement artifacts and significant damage to both sensors and the sensing system. In fact, the effects of nuclear environments on this technology are not yet well addressed and understood. The nuclear radiation on SHM systems with PWAS exposed to irradiation effects has been tested preliminarily. However, the analytical model of PWAS based sensor and sensing system has not been developed with adequate solutions and guideline. It is of paramount importance to assess and understand how the piezoelectric ultrasonic SHM systems are affected by nuclear radiation and high temperature factors; thus to protect them when necessary.

This paper addresses the issue of analytical modeling of nuclear environmental effects in PWAS SHM system. Our focus is mainly of PVP application in the DCSS system. This paper discusses some of the challenges associated with the nuclear effects on DCSS system. Fundamental research including the transition of SHM technologies to PVP applications is discussed.

\section{DRY CASK STORAGE SYSTEM SAFETY ASSESSMENT NEED}

Following the issuance of the Blue Ribbon Commission (BRC) on America's Nuclear Future Final Report in 2012, interim storage of spent nuclear fuel from reactor sites has gained additional importance and urgency for resolving wastemanagement-related technical issues. In total, there are over 1482 dry cask storage system (DCSS) in use at US plants, storing 57,807 fuel assemblies. On July 12, 2011, the Nuclear Regulatory Commission (NRC) issued recommendations on how to enhance "spent fuel makeup capability and instrumentation for the spent fuel pool". This includes the recommendation to provide sufficient safety-related instrumentation (able to withstand design basis natural phenomena) to monitor from a control room the key parameters of the spent fuel pool (e.g., temperature, radiation level, etc.). Monitoring has been identified by DOE as a high priority crosscutting need. Monitoring is necessary to determine and predict the degradation state of the systems, structures, and components (SSCs) important to safety (ITS) and is required by regulation (10 CFR 72.122 and 10 CFR 72.128). Revisions to NUREG 1927 suggest requirements for monitoring and inspection of dry storage systems as part of aging management plans. To ensure that nuclear power remains clean energy, safe, long-term management of used nuclear fuel and high level radioactive waste "remains a national priority".

Therefore, nondestructive structural material degradation and condition monitoring is in urgent need and must be integrated into the fuel cycle to quantify the "state of health", and more importantly, to guarantee the safe operation of existing nuclear power plants (NPP) and radioactive waste storage systems (RWSS) during their predicted life span. Innovative approaches are desired to evaluate degradation and aging phenomena of used fuel containers and storage facilities under extended storage. To meet the ever-growing awareness of nuclear safety, a state-of-the-art nuclear structural health monitoring (N-SHM) system is necessary that uses in-situ sensing technologies to monitor material degradation and aging for DCSS canister and similar structures (e.g., wet storage pools), as conceptually illustrated in Figure 1.

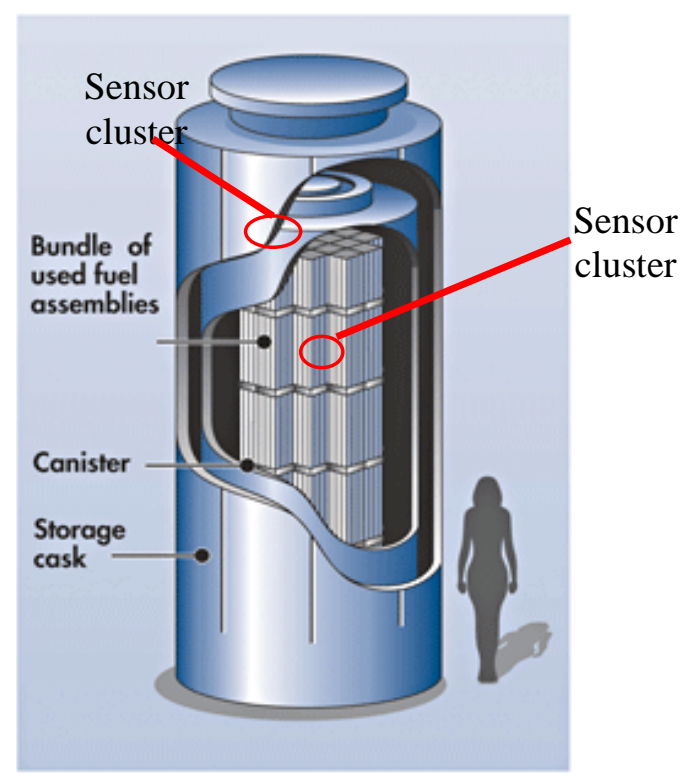

Fig 1: Sensor cluster on typical dry cask storage system (http://www.nrc.gov/waste/spent-fuel-storage/diagram-typicaldry-cask-system.html) 


\section{PIEZOELECTRIC WAFER ACTIVE SENSORS}

The key technology to an effective N-SHM system is the sensing element that can detect the degradation under the harsh nuclear DCSS environment. The past two decades have witnessed an extensive development of SHM sensor technology [3]-[5]. A wide range of sensors have been developed particularly for generating and receiving acousto-ultrasonic waves. Piezoelectric wafer active sensors (PWAS) have emerged as one of the major SHM technologies. A variety of damage detection methods can be applied to this type of sensor [3]: (a) propagating ultrasonic waves, both acoustic emission (AE) and guided ultrasonic waves (GUW); and (b) standing ultrasonic waves, i.e., electromechanical impedance spectroscopy (EMIS) as illustrate in Fig 2 and Fig 3.

- $\mathrm{AE}$ for crack initiation has been shown to enable the detection of crack initiation and crack progression; $\mathrm{AE}$ provides earlier warning of impending damage than any other methods. Because it is very sensitive to damage events, the $\mathrm{AE}$ method has been used for many applications in aerospace and civil engineering applications.

- GUW quantitative damage detection and evaluation relies on in interrogative ultrasonic waves propagating and reflecting within the structure to identify wave field disturbances due structural damage and flaws. An N-SHM system using interrogative GUW would be able to cover large areas from one single location, thereby being cost-effective and time-efficient. Research on embedded PWAS GUW-SHM has been conducted nationally and internationally for damage detection on both metallic and composite thin-walled structures.

- EMIS for local material degradation monitoring: is considered a promising approach for PWAS structural NDE. This method utilizes high frequency structural excitations, which are typically higher than $30 \mathrm{kHz}$ through surface-bonded PWAS to monitor changes in the structural E/M impedance. Previous studies have confirmed that EMIS is sensitive to very small amounts of material changes, suggesting that EMIS offers the potential for detection of the progression of small damage at the material-level in a metallic material.

Our previous research has successfully demonstrated the use of PWAS on thick steel components [19] and investigated the durability and survivability of the PWAS transducers under various exposures (cryogenic and high temperature, temperature cycling, freeze-thaw, outdoor environment, operational fluids, large strains, fatigue load cycling) [6]. In most cases, the PWAS survived the tests successfully. The cases when the PWAS did not survive the tests were closely examined and possible cause of failure was discussed. The test results indicated that PZT PWAS can be successfully used in cryogenic environment; however, it does not seem to be a good candidate for high temperature. Repeated differential thermal expansion and extended environmental attacks can lead to
PWAS failure. This emphasizes the importance of achieving the proper design of the adhesive bond between the PWAS and the structure, and of using protective coating to minimize the ingression of adverse agents. Our preliminary work on the effects of nuclear radiation on PWAS exposed to irradiation effects published in 2012 [7]. We have discovered that the short term irradiation affected the capacitance and the electromechanical impedance of PWAS. A predictive modeling on PWAS under radiation effect for DCSS system is necessary.

Propagating Lamb waves

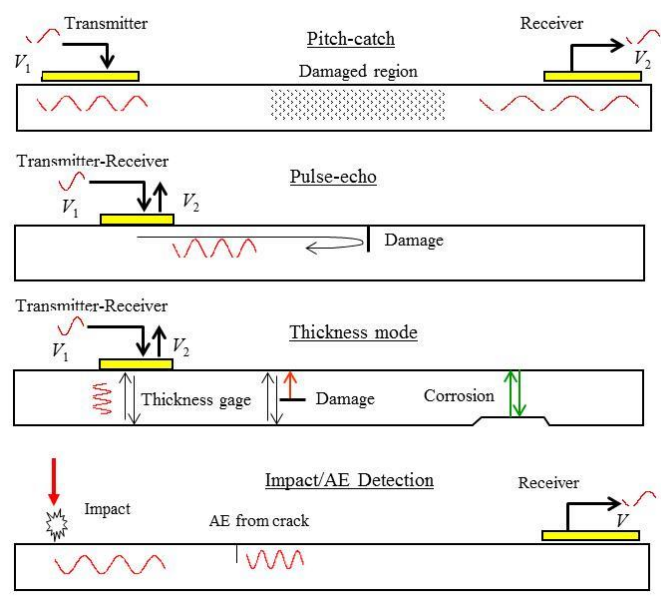

Fig 2: The PWAS are used for structural sensing with propagating ultrasonic guided waves. The propagating wave methods include: pitch-catch; pulse-echo; thickness mode; and passive detection of impacts and acoustic emission (AE).

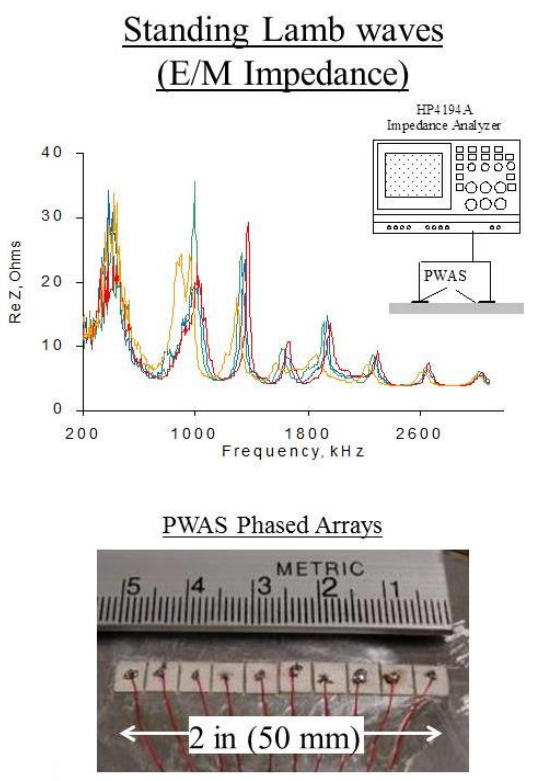

Fig 3: The PWAS are used for structural sensing includes standing waves and phased arrays. 


\section{GUIDED WAVE MODEL WITH NUCLEAR EFFECTS}

Environmental variability of a Nuclear-SHM system includes changes in both the sensors and the sensing methodology (AE, GUW, and EMIS). In this paper, the analytical model of nuclear effects is focused on analytical modeling of PWAS bonded on the steel structure used in the canisters. We developed the ultrasonic modeling with nuclear effects include: (a) analytical codes for AE and GUW propagation in metallic structures; (b) analytical modeling of the EMIS method on 1-D and 2-D structures.

We performed multi-physics predictive simulation of the generation, propagation, and reception of guided ultrasonic waves using PWAS transducers in thicker steel shells as well as in reinforced concrete media. The advantage of predictive simulation is that it allows inexpensive exploration of various parameters (plate thickness, geometry, crack size, corrosion size, PWAS characteristics, etc.) Our goal is to use predictive simulation to explore a wide parameter space and identify the most successful candidate for actual experimental testing.

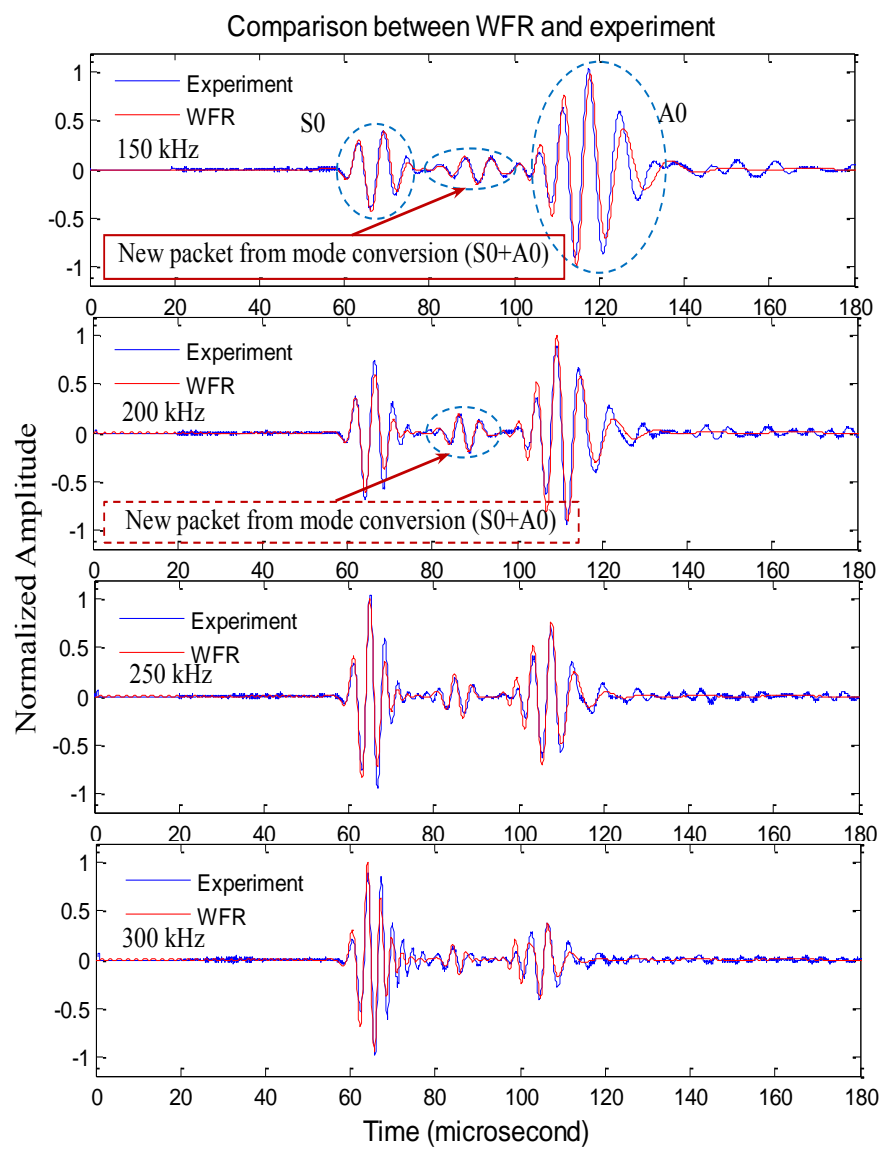

Fig 4: Comparison between WFR simulations and experiments for GUW interaction with a notch

In previous research, we have developed an analytical codes "Wave Form Revealer" (WFR) for 1-D wave propagation with damage interactions on metallic structures [8]. The software program WFR is a Matlab graphical user interface (GUI) environment to predict the waveform of the analytical modeling for SHM. This software allows users to get the desired analytical solution by inputting material properties, specimen geometry, excitation signal count number, excitation signal frequency, and time range. Fig 4 shows the WFR simulation results of GUW propagation at $150 \mathrm{kHz}$ on a $3.17-\mathrm{mm}$ Aluminum plate where a notch is later added to simulate "damage" compared with experimental measurements. The analytical waveforms agree well with experimental data.

In this paper, we extended the modeling tools to address the ultrasonic wave modeling on thick steel structure used in DCSS. Our aim is to identify and quantify the possible influences of the nuclear environment typical of DCSS (temperature and radiation) to the PWAS based sensor and sensing system, and to develop adequate solutions and guidelines accordingly. The analytical modeling of the pitchcatch process between two PWAS transducers separated by a distance $x$ was carried out in frequency domain in four steps [1,2]: (i) Fourier transform the time-domain excitation signal $V_{e}(t)$ taken into the frequency domain spectrum, $\tilde{V}_{e}(\omega)$; (ii) Calculate the frequency-domain structural transfer function at the receiver location, $G(x, \omega)$; (iii) Multiply the structural transfer function by frequency-domain excitation signal to obtain the frequency domain signal at the receiver, i.e., $\tilde{V}_{r}(x, \omega)=G(x, \omega) \cdot \tilde{V}_{e}(\omega)$; (iv)Perform inverse Fourier transform to obtain the time-domain receiver signal,

$$
V_{r}(x, t)=\operatorname{IFFT}\left\{\tilde{V}_{r}(x, \omega)\right\}=\operatorname{IFFT}\left\{G(x, \omega) \cdot \tilde{V}_{e}(\omega)\right\}
$$

Environmental variability includes the effect of nuclear environmental changes on PWAS and also on the ultrasonics waves. Environmental variability includes the effect of environmental temperature changes and radiations. For example, the guided waves are functions of temperature through the elastic modulus and density in the dispersion equations,. The elasticity of metal depends on its temperature. According to published data [9] the relationship between Young's modulus and the temperature of aluminum can be represented as

$$
E=-3.9 e^{-0.0033 T}+79
$$

where $E$ is Young's Modulus in GPa, and $T$ is temperature in Kelvin. We found that the temperature effect is principally due to the modification of the elastic modulus, $E$, rather than the geometrical expansion contraction because the ultrasonic waves depend on both density $\rho$ and modulus $E$. The specialized literature indicates that the elasticity modulus $E$ of metals varies strongly with temperature. Using our WFR software, we presented that the temperature effect is majorly due to the modification of the Young modulus instead of the geometrical expansion/contraction of the material as shown in Fig 5. 

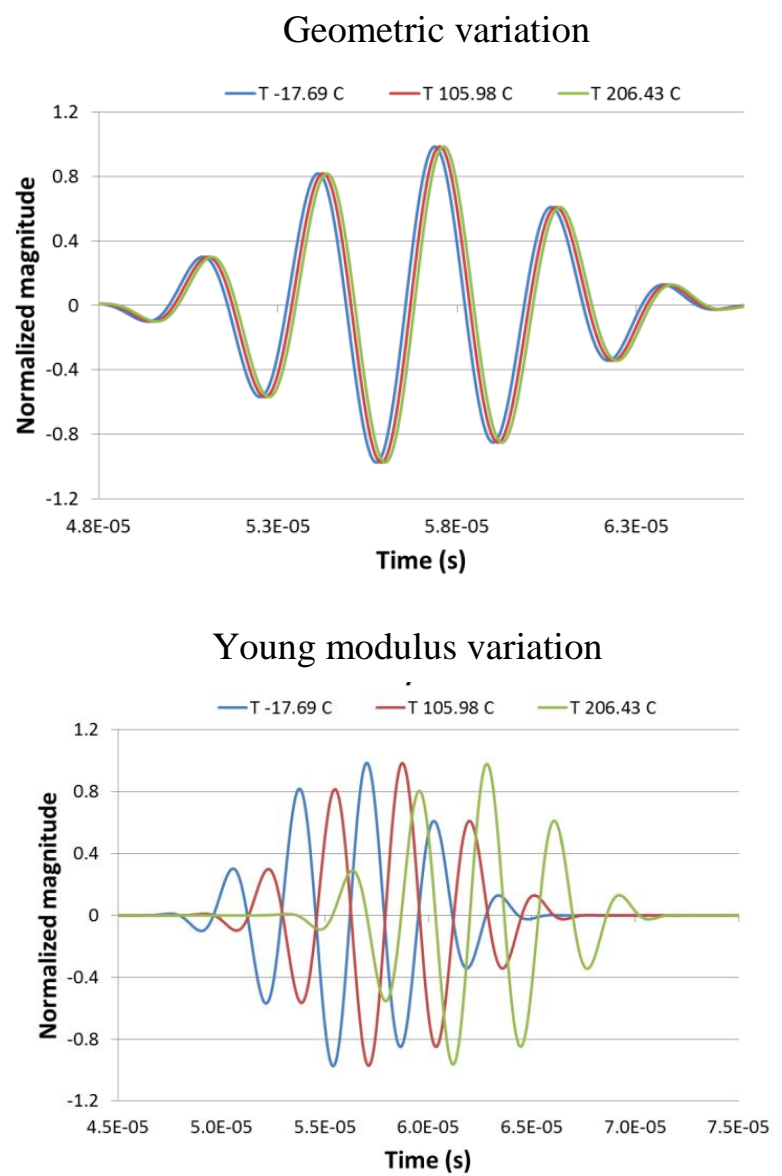

Fig 5: Temperature effects on geometry and Young's modulus

\section{ELECTROMECHANICAL IMPEDANCE MODEL}

For a linear piezoelectric material, the relation between the electrical and the mechanical variables can be described by linear relations:

$$
\left[\begin{array}{l}
S \\
D
\end{array}\right]=\left[\begin{array}{ll}
s^{E} & d_{t} \\
d & \varepsilon^{T}
\end{array}\right]\left[\begin{array}{l}
T \\
E
\end{array}\right]
$$

where $S$ is the mechanical strain; $T$ is the mechanical stress; $E$ is the electric field; $D$ is the charge density; $s$ is the mechanical compliance; $d$ is the piezoelectric strain constant; and $\varepsilon$ is the permittivity. The superscripts $E$ and $T$ indicate that those quantities are measured with electrodes connected together and zero stress, respectively, and the subscript $t$ indicates transpose. The first equation describes the converse piezoelectric effect and the second describes the direct effect.

The principles of electro-mechanical (E/M) impedance method are illustrated in Fig 6. The drive-point impedance presented by the structure to the active sensor can be expressed as the frequency dependent variable

$$
Z_{s t r}(\omega)=k_{s t r}(\omega) / j \omega=k_{e}(\omega)-\omega_{m}^{2}(\omega)+j \omega c_{e}(\omega) .
$$

Through the mechanical coupling between PWAS and the host structure, on one hand, and through the E/M transduction inside the PWAS, on the other hand, the drive-point structural impedance is reflected directly in the electrical impedance, $Z(\omega)$, at the PWAS terminals

$$
Z(\omega)=\left[j \omega C\left(1-\kappa_{31}^{2} \frac{\chi(\omega)}{1+\chi(\omega)}\right)\right]^{-1}
$$

where $\mathrm{C}$ is the zero-load capacitance of the PWAS and $\kappa_{31}$ is the E/M cross coupling coefficient of the PWAS $\left(\kappa_{31}=d_{31} / \sqrt{\overline{s_{11} \bar{\varepsilon}_{33}}}\right)$, and $\chi(\omega)=k_{s t r} / k_{P W A S}$ with $k_{P W A S}$ being the static stiffness of the PWAS.

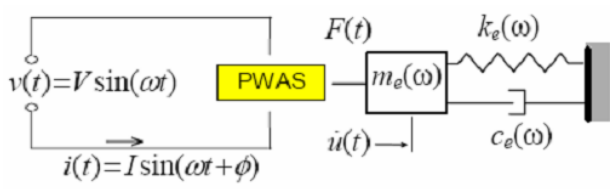

Fig 6: E/M coupling between PWAS and structure for a 1-D dynamic model

\section{EMIS Analytical Modeling}

There are several analytical methods to calculate the EMIS of PWAS. Normal mode expansion (NME) was used for simple structures. Giurgiutiu and Zagrai [16] gave the detailed derivation of complex formulas for admittance and impedance of piezoelectric bar. Later they considered PWAS radial mode vibration of a thin disk [17]. The 2-D analysis of PWAS impedance for circular-crested Lamb waves were performed in cylindrical coordinates using the Bessel functions formulation.

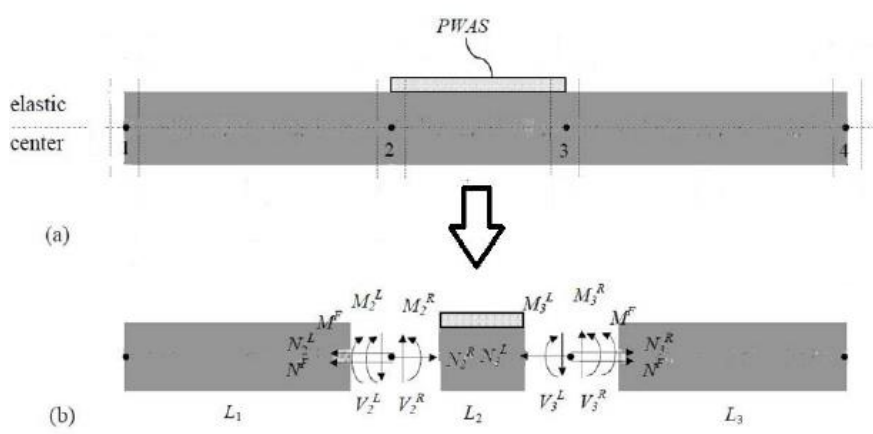

Fig 7: Free vibration of a uniform beam: (a) schematic of beam elements; (b) free-body diagram.

The transfer matrix method (TMM) is an analytical method for structural analysis which is alternative to modal expansion. The TMM is a way to determine the natural frequencies of a non-uniform beam. The TMM principle is to break up the structure into smaller components across which one calculates the transfer of a state vector (displacements and forces). For example, if a beam with a PWAS is bonded to the structure as per Fig 7a, by applying TMM, the beam can be separated into segments with the same configuration (length, width and excitation) as per Fig 7b. Cuc [18] expanded the frequency resonances of a uniform beam from the range of $1-30 \mathrm{kHz}$ to a 
higher frequency range $10-500 \mathrm{kHz}$ and performed the reliability and accuracy analysis of all available theoretical models given experimental data for the uniform beam specimen.

\section{EMIS Finite Element Modeling}

A free PWAS has been modeled in order to have a fundamental understanding of the multi-physics based modeling approach and its efficiency using ANSYS multi-physics software with the implicit solver in the frequency domain. To perform the coupled-field analysis of PWAS transducers, we used coupledfield elements, which could deal with both mechanical and electrical fields. In the physics based coupled-field analysis of piezoelectric materials, the stress field and the electric field are coupled to each other such that change in one field will induce change in the other field. The coupled-field finite elements used in our analysis are the brick elements (SOLID5, SOLID226) that have 8 or 20 nodes with up to six degrees of freedom (DOF) at each node. When used for piezoelectric analysis, an additional DOF, the electric voltage can be added in addition to the displacement DOF's. Reaction forces FX, FY, FZ correspond to the X, Y, Z displacement DOF's, respectively. The electrical charge $\mathrm{Q}$ is the electrical reaction corresponding to the voltage DOF. The charge $\mathrm{Q}$ is then used to calculate the admittance and impedance data. The admittance $\mathrm{Y}$ is calculated as $\mathrm{I} / \mathrm{V}$, where $\mathrm{I}$ is the current in ampere and $\mathrm{V}$ is the applied potential voltage in volts. The current comes from the charge accumulated on the PWAS surface electrodes and is calculated as $I=j \omega \sum Q_{i}$ with $\omega$ being the operating frequency, $j$ is the complex number and $\sum Q_{i}$ is the summed nodal charge. In this study, the PWAS was modeled using the 3D MP-FEM approach with SOLID5 and SOLID226 elements. SOLID5 is a coupledfield brick with eight nodes and up to six DOF per node while SOLID226 has twenty nodes with up to four DOF per node. A free square-shaped PWAS of dimension $7 \times 7 \times 0.2 \mathrm{~mm}^{3}$ was modeled. The APC-850 material properties were assigned to the PWAS as

$$
\begin{gathered}
C_{p}=\left[\begin{array}{cccccc}
97 & 49 & 49 & 0 & 0 & 0 \\
49 & 97 & 44 & 0 & 0 & 0 \\
49 & 49 & 84 & 0 & 0 & 0 \\
0 & 0 & 0 & 24 & 0 & 0 \\
0 & 0 & 0 & 0 & 22 & 0 \\
0 & 0 & 0 & 0 & 0 & 22
\end{array}\right] \mathrm{GPa} \\
{\left[\varepsilon_{p}\right]=\left[\begin{array}{cccc}
947 & 0 & 0 \\
0 & 605 & 0 \\
0 & 0 & 947
\end{array}\right] \times 10^{-8} \mathrm{~F} / \mathrm{m}} \\
{\left[e_{p}\right]=\left[\begin{array}{cccccc}
0 & 0 & 0 & 0 & 12.84 & 0 \\
0 & 0 & 0 & 12.84 & 0 & 0 \\
-8.02 & -8.02 & 18.31 & 0 & 0 & 0
\end{array}\right] \mathrm{C} / \mathrm{m}^{2}}
\end{gathered}
$$

where $\left[C_{p}\right]$ is the stiffness matrix, $\left[\varepsilon_{p}\right]$ is the dielectric matrix and $\left[e_{p}\right]$ is the piezoelectric matrix. The density of the PWAS material is assumed to be $\rho=7600 \mathrm{~kg} / \mathrm{m}^{3}$.

Spectra from simulation and experiment of the free-PWAS are sweeping from 1 to $1000 \mathrm{kHz}$ are presented in Fig 8 . Globally, good matching is observed, but some slight differences are visible especially for the third resonance around $\sim 500 \mathrm{kHz}$. Small differences are expected in the numerical response when compared to the experimental impedance because the wiring was not modeled and unavoidable and measuring errors may also occur. Nonetheless, this comparison illustrates that good agreement can be obtained when MP-FEM method is used, which is an important improvement over the use of the conventional FEM method or the analytical models.

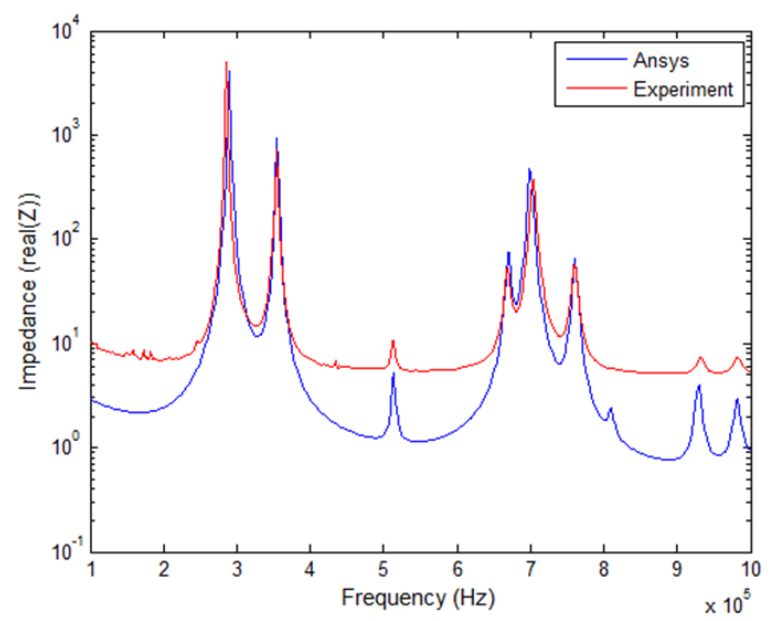

Fig 8: PWAS EMIS obtained from MP-FEM and measured from HP4194 equipment, respectively.

\section{POTENTIAL WORK ON ANALYTICAL MODEL}

The implementation of SHM for DCSS and similar PVP systems will require additional research and development as well validation as an effective nondestructive testing (NDT) and nondestructive evaluation (NDE) tool. As existing DCSS systems continue to operate, some of them exceeding their designed life span, new degradation processes are being found. For these new degradation processes, the effectiveness of existing inspection and NDE tools is unknown [15]. Many inspections cannot be conducted effectively on a number of materials and configurations. Potential radiation exposure and obstructed areas also limit the inspectability of such systems. Other conditions found in the field that may limit the inspectability include surface condition in the inner and outer diameters of weldments, access constraints, and tapers that exist on components transitioning from one diameter to another. For these reasons, new ways to assess the in-service state of structures must be found. A potential approach is to use SHM concepts and technologies that have been developed elsewhere but may be quite applicable to PVP systems with appropriate modifications. 
The harsh environment associated with PVP systems will challenge the use of many types of sensors due to high temperature and/or gamma radiation stressors. For applications to DCSS the development of appropriate sensors that can tolerate initial cask loading temperatures and radiation levels with minimal functional degradation is necessary. This can be addressed through either the design or modification of materials to increase resistance to the effects of radiation and temperature or developing new materials or sensing schemes. The LAMSS team at USC has begun testing PWAS systems under radiation field [13]: Durability and survivability of PWAS transducers were tested under gamma ray exposure. A Co-60 gamma source was used to irradiate a set of PWAS transducers in an irradiator with different exposure times. The dose rate and total absorbed dose were calculated using Monte Carlo simulations (MCNPX code). The PWAS material properties, electrical contact change were characterized through a series of tests. The electromechanical impedance spectrum of PWAS was measured. This study provides a first step towards the fundamental understanding of the PWAS irradiation survivability. Another outcome of this study is to evaluate the potential of PWAS transducers as irradiation sensors for nuclear applications.

After these preliminary results, we studied the effect of the variations of the PWAS mechanical behavior, the dielectric matrix, and the piezoelectric matrix in order to better understand and to predict the effect of the radiation on these different components using this multi-physics finite element method. Three variability effects will be considered: (i) the sensory system, (ii) the structural components, and (iii) the nuclear environmental conditions (temperature and radiation). The temperature and radiation effects will be examined through parameter studies. Eventually, the analytical tools developed in this subtask will permit fast and efficient evaluation of the effect of variability on the structural sensing process.

(a) Sensory system variability considers the effects of sensor aging and degradation. We will extend our preliminary results to a full parametric study (size, density, stiffness matrix, dielectric matrix, piezoelectric matrix, etc.). The effects of variability in PWAS positions on the structure and of impedance coupling between PWAS and structure will also be assessed.

(b) Structural variability includes the effect of slight variations in material properties and/or the structural geometry and tolerances. We will first determine sensitivity of individual sensing methods. Then we will introduce damage and repeat the variability study to see if damage can still be detected in the presence of structural variability.

(c) Environmental variability considers the effects of temperature changes and radiation. We will superpose these changes on the analytical models and determine how much the structural sensing signals change is due to these environment effects. We will establish the sensitivity of various structural sensing methods to environmental disturbances. Results from subtask 2.2 will be used to update the model parameters to further study the environmental variability

\section{SUMMARY AND CONCLUSIONS}

SHM is a multidisciplinary process that involves several disciplines that must be closely coordinated. Sensors are being used to measure parameters such as temperature, pressure, radiation levels, $\mathrm{pH}$, and wall thickness or to indicate that damage or failure in a system has already occurred (i.e. detection of a leak). But through the implementation of SHM approach the detection of materials degradation at relatively early stages, before the damage occurs, can be potentially achieved. Therefore, the development of active and passive nondestructive evaluation methods based on SHM provides an opportunity to progress the capability of monitoring DCSS and similar PVP systems. The integration of SHM with existent NDE tools in nuclear fields can increase the confidence of the safe operation and provide assurance of in-service reliability. Moreover, the development of SHM technologies can minimize human intervention, decrease the cost associated with NPP operation, and improve the reliability of essential systems by continuously assessing the structural integrity of nuclear related facilities. While SHM have been employed in different fields, its applicability for PVP systems will require further development and evaluation. This will required research to address some of the challenges that were discussed. SHM monitoring can provide decision makers, regulatory agencies, and PVP system operators with timely information on the health of the system. Ultimately, this information will result in the reduction of operation and maintenance cost, and the timely response can protect civilian population from catastrophic system failure.

We address the fundamental issue of analytical modeling of nuclear environmental effects in PWAS N-SHM system for steel canister in DCSS in the present study. Some of the challenges associated with the nuclear effects on DCSS system are also discussed. We studied various nuclear effects on the guided wave propagation as well as electromechanical impedance through both analytical and finite element modeling. We found that the temperature effect is principally due to the modification of the elastic modulus, $\mathrm{E}$, rather than the geometrical expansion contraction. This preliminary work provides the guidance for the development of PWAS based NSHM.

\section{ACKNOWLEDGEMENT}

The authors would like to acknowledge the financial support from Department of Energy award DE-NE0000726 with program manager Mr. Kenny Osborne. 


\section{REFERENCE}

[1] NRC 10 CFR: US Nuclear Regulatory Commission Regulations: Title 10, Code of Federal Regulations, Part 72: Licensing requirements for the independent storage of spent nuclear fuel and high-level radioactive waste, and reactorrelated greater than Class $\mathrm{C}$ waste, http://www.nrc.gov/reading-rm/doc-collections/cfr/ accessed Jan 2014

[2] Nureg-1927, Standard review plan for renewal of spent fuel dry cast storage system licenses and certificates of compliance - final report, http://www.nrc.gov/readingrm/doc-collections/nuregs/staff/sr1927/ Mar, 2011

[3] Giurgiutiu, V; Gresil, M; Lin, B; Cuc, A; Shen, Y; Roman, C (2012) "Predictive Modeling of Piezoelectric Wafer Active Sensors Interaction with High-frequency Structural Waves and Vibration", Acta Mechanica, March 2012, No. 223, pp. 1681-1691

[4] Balageas, D. et al., (eds.), Structural Health Monitoring, ISTE (2006).

[5] Alleyne, D.N. "The Nondestructive Testing of Plates Using Lamb Waves", Mechanical Engineering Department, Imperial College of London, London, (1991).

[6] Lin, B.; Giurgiutiu, V.; Pollock, P.; Xu, B.; Doane, J.; (2010) "Durability and Survivability of Piezoelectric Wafer Active Sensors on Metallic Structures", AIAA Journal, Vol. 48, No. 3, pp. 635-643, March 2010A The

[7] Lin, B.; Mendez-Torres, A. E.; Gresil, M.; Giurgiutiu, V. (2012) "Structural Health Monitoring with Piezoelectric Wafer Active Sensors Exposed to Irradiation Effects", ASME 2012 Pressure Vessels and Piping Division Conference, 1519 July 2012, Toronto, Ontario, Canada, paper PVP201278848

[8] Shen, Y.; Giurgiutiu, V. (2014) "WaveFormRevealer - An Analytical Framework and Predictive Tool for the Simulation of Multimodal Guided Wave Propagation and Interaction with Damage", Structural Health Monitoring - an International Journal. (accepted)

[9] Young Modulus of Elasticity for Metals and Alloys http://www.engineeringtoolbox.com/young-modulusd 773.html.

[10] Gresil, M. et al., "Predictive Modeling of Space Structures for SHM with PWAS Transducers," Book Predictive modeling of space structures for SHM with PWAS transducers, 5190, 2011.

[11] Andreaus, U. et al., "Non-linear dynamics of a cracked cantilever beam under harmonic excitation," International Journal of Non-linear Mechanics, vol. 42, 2007, pp. 566-575.

[12] Kamal, A.; Lin, B.; Giurgiutiu, V. (2013) "Exact analytical modeling of power and energy for multimode lamb waves excited by piezoelectric wafer active sensors", Journal of Intelligent Material Systems and Structures, Vol. 25, No. 4, pp. $452-471$

[13] Lin, B.; Mendez-Torres, A. E.; Gresil, M.; Giurgiutiu, V. (2012) "Structural Health Monitoring with Piezoelectric Wafer Active Sensors Exposed to Irradiation Effects", ASME 2012 Pressure Vessels and Piping Division Conference, 1519 July 2012, Toronto, Ontario, Canada, paper PVP201278848

[14] Giurgiutiu, V. (2008) Structural Health Monitoring with Piezoelectric Wafer Active Sensors, Elsevier Academic Press, 760 pages, ISBN 978-0120887606, 2008
[15] Doctor, S.R. (2007), "Nuclear Power Plants NDE Challenges: Past, Present and Future", Review of Quantitative Nondestructive Evaluation, Vol. 26, 2007

[16] Giurgiutiu, V and Zagrai, A.N. (2000) "Characterization of Piezoelectric Wafer Active Sensors", Journal of Intelligent Material Systems and Structures, Vol. 11, No. 12, December 2000, pp. 959-976

[17] Giurgiutiu, V.; Zagrai, A. N. (2002) "Embedded Self-Sensing Piezoelectric Active Sensors for Online Structural Identification", ASME Journal of Vibration and Acoustics, Vol. 124, No. 1, January 2002, pp. 116-125

[18] Cuc, Adrian (Ph.D. 2010) Structural Health Monitoring of Adhesively Bonded Joints with Piezoelectric Wafer Active Sensors (2010) Theses and Dissertations. Paper 329.http://scholarcommons.sc.edu/etd/329

[19] Yu, L.; Momeni, S.; Godinez, V.; Giurgiutiu, V.; Ziehl, P.; $\mathrm{Yu}$, J. (2012) "Dual Mode Sensing with Low-Profile Piezoelectric Thin Wafer Sensors for Steel Bridge Crack Detection and Diagnosis", Advances in Civil Engineering, Vol. 2012, Article ID 402179, 2012, doi:10.1155/2012/402179 